\title{
手征模型中的 $\boldsymbol{H}$-变换、无穷多非定域守 恒流的 Noether 分析与 Kac-Moody 代数结构及其推广
}

\author{
乔玲丽 \\ 吴聇时

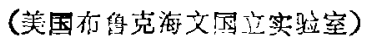 \\ (中国科学院理论物理研究所, 北京) \\ 侯伯宇 \\ 葛墨林 \\ (西北大学, 西安) \\ (兰州大学)
}

\section{摘 要}

本文通过引入局域化的 $H$-变换,对手征模型进行了系统的研究. 指出了: 在主 手征模型中无穷多非定域守恒流可以作为 Noether 流由 $H$-变换自然导出，并可写 出各阶流的系统方案。在光锥坐标下，直接证明存在明显的 Kac-Moody 代数结构, 并 阐明了这种结构与 $H$-变换的关系。文中还将上述结果推广到自对偶杨-Mills 场, 得 出的结论是: 上述结果仍然有效，而且又找出了 $H$-变换与 Bäcklund 变换之间的内 在联系。

\section{一、前 言}

近作来,在两维手征 (Chiral) 模型中的无穷多提流的讨论方面, 有相当大的发展 ${ }^{[1-6]}$. 平始, 人们从运䢵方程出发, 引入了线性化方程 ${ }^{[7]}$, 从而使得一系列守恒流的研究系统化. 从 经验中知道, 守恒量可能与桨种变换性质有关, 那么与无穷多守恒流相联系的这种尚未被揭示 出来的“隐藏”的对称性是什么呢？换言之，无穷多守恒流如何作为一种与某种变换性质椙联 系的 Noether 流很自然地导出? 这是个很有兴趣的问题.

文献 [8]曾经部分地讨论过这个问题。从拉氏函数出发, Dolan 和 Roos 认为如果无穷多 守恒流是一种 Noether 流, 那么它们应当与j一种 off-shell (不代入运动方程) 的对称性相轵系; 他们还写出了前两阶流的具体形式, 当它们在 on-shell (代入运动方程) 时, 就是通常的守恒 流. 但是, 他们并没有用一种系统的方法写出任意阶流的形式,而且由于所有的讨论并未与楳 种明显的变换性质相联系, 所以实际上并没有揭示出“隐藏”对称性的实质.

与此平行的另一个重要的问题是: 关于无穷多守恒流的讨论中的代数结构问题[8,9], 即所 谓 Kac-Moody 代数问题. 如果我们找到一种机制将代数结构与变换性质联系起来, 将是非常 
有兴趣的问题.

本文在总结了过去这方面的一系列工作结果 ${ }^{[10-12]}$ 的基础上,提出下面的看法.

1. 以主手征模型为例,对场变量引入一种变换, 以后称之为 $H$-变换. 在 $H$-变换中包括含 有任意参数 $l$ 的么正矩阵 $U$, 当 $U$ 满足通常的线性方程时，在 $H$-变换下整个作用量并不改 变, 亦即可使运动方程不变. 于是从这种不变性出发, 可以得到参数化的守恒流, 从而得到 ofi-shell 对称性与无穷多非定域流之间的联系,并可写出各阶非定域流的方案.

2. 从上述参数化的变换性质出发，直接推得通常定义的算子间的 Kac-Moody 代数关 系 ${ }^{[8,11]}$. 这里将会看到所用的方法与文献 [9]相比,不但明显地揭示出 Kac-Moody 代数结构与 参数化变换的内在联系, 而且.更易推广到其它许多情况, 同时比文献[11]更为严格.

3. 将上述思想应用于自对偶杨-Mills（SL(N,C) 情况) 场中去, 得到了同样的代数关系, 更有趣的是：同时又揭示出参数化变换与通常的 Bäcklund 变换之间的关系.

下面除了杨-Mills 场的情况之外,计算是在二维闵氏时空中进行的，所用到的度规是 $\eta_{00}=-\eta_{11}=1$ 以及 $a_{01}=-e_{10}, e^{01}=-6^{10}=-1$. 当然所有的计算将会完无困难地推广到 欧区空间中去.

\section{二、H-变换与参数化非定域流的 Noether 分析}

现在讨论无穷多非定域守恒流与场变量的变换性质之间的联系，并招此提出一系列守恒 流的系统方案.

在主手征模型，拉氏函数密度为:

$$
\mathscr{L}(x)=\frac{1}{4} T_{r}\left\{\partial_{\mu} g(x) \partial^{\mu} g^{-1}(x)\right\},
$$

其中 $g(x)$ 属于群 $G$. 定义

运动方程遂为:

$$
A_{\mu}(x)=g^{-1}(x) \partial_{\mu} g(x),
$$

显然， $A_{\mu}$ 满足平直条件:

$$
\partial^{\mu} A_{\mu}=0 \text {. }
$$

$$
\partial_{\mu} A_{\nu}-\partial_{\nu} A_{\mu}+\left[. A_{\mu}, A_{\nu}\right]=0 .
$$

通常, 对场量 $g$ 可以作整体变换

$$
\delta g=-g T,
$$

其中 $T=T_{\rho} \alpha^{a}, \alpha^{a}$ 为无穷小参数, $T^{a}$ 为群 $G$ 生成元, 是反厄米的. 在变换 (2.5) 式, 有

$$
\delta \mathscr{L}=-\frac{1}{2} T_{r}\left\{A_{\mu} \partial^{\mu}\left(g^{-1} \delta g\right)\right\}=0 .
$$

但是如果将上述常数矩阵 $T$ 变成局域化的矩阵 $\Lambda(x)$, 并使之仅相差一局域么正转动

$$
T \rightarrow \Lambda(x)=U(l, x) T U^{-1}(l, x),
$$

其中 $l$ 为任意参数,那么此时的 $\delta \mathscr{L}$ 将不再为零. 从(2.7)式我们看到, $\Lambda(x)$ 与 $T$ 的不同实 质上在于规定了新的“平行”的定义，对此点的理解，在过去讨论磁单极的工作中 ${ }^{[13]}$ 已有讨论， 这里不再鏊述. 直到现在, 我们并未对 $(2.7)$ 式中的 $U(l, x)$ 作任何限制. 作为一和特珠的选 
择, 我们令 $U$ 满足反政射方程

$$
\begin{aligned}
& \partial_{0} U=\frac{l}{1-l^{2}}\left(l A_{0}-A_{1}\right) U, \\
& \partial_{1} U=\frac{l}{1-l^{2}}\left(l A_{1}-A_{0}\right) U .
\end{aligned}
$$

如果变换 (2.7)式中的 $U$ 满足 (2.8)与(2.9)式中任何一个时称为 off-shell 的 $H$-变换, 如界变换 (2.7)式中的 $U$ 满足(2.8)! 年(2.9)式两者时称为 on-shell 的 $H$-变换. 这是因为(2.8)与(2.9)式 的可积条件将导致运动方程与平直条件. 于是变换 (2.5) 式为:

$$
\delta g=-g \Lambda(x)=-g U(l, x) T U^{-1}(l, x) .
$$

如果我们不代入运动方程时, 将使用 off-shell 的 $H$-变换, 记为 Off-SHT, 如果代入运动 方程时,将使用 on-shell 的 $H$-变换记为 On-SHT.

应当指出, 在 off-SHT 情况下, $\mathscr{L}$ 仍仅改变一个全散度, 亦即不改变作用量一一在 cn-shell 时将保持运动方程不变. 从(2.8)式可得

$$
U(l, x)=P \exp \left\{\frac{l}{1-l^{2}} \int_{-\infty}^{x} d y\left(l A_{1}(y, t)-A_{0}(y, t)\right)\right\} .
$$

而在讨论守恒流时, 又必须回到 on-shell 的情况, 此时有

$$
\delta \mathscr{L}=\frac{1}{2} \partial^{\mu} T_{r}\left(U^{-1} A_{\mu} U T\right) .
$$

生讨论 off-shell 情况下的 $\delta \mathscr{L}$ 时, 我们将(2.9)式代人(2.10)式, 并出此计算 $\mathscr{L}$ 的变 更, 由

$$
\delta \mathscr{L}=\frac{1}{2} T_{r}\left\{\left[U^{-1} A_{0} U, U^{-1} \partial_{0} U\right] T-\left[U^{-1} A_{1} U, U^{-1} \partial_{1} U\right]\right\}
$$

有

$$
\begin{aligned}
\delta \mathscr{L}= & \frac{1}{2} T_{r}\left\{\frac{1-l^{2}}{l}\left\lfloor U^{-1} \partial_{0} U, U^{-1} \partial_{1} U\right] T^{+}+l\left[U^{-1} A_{1} U, U^{-1} \partial_{0} U\right] T\right. \\
& \left.+l\left[U^{-1} A_{1} U, U^{-1} A_{0} U\right] T+l\left[U^{-1} \partial_{1} U, U^{-1} A_{0} U\right] T\right\} .
\end{aligned}
$$

考虑(2.4)式, 经过计算, 得

$$
\delta \mathscr{L}=\frac{1}{2} \partial_{\mu} T_{r}\left\{\left(\frac{1-l^{2}}{l} \sigma^{\mu \nu} U^{-1} \partial_{\nu} U-l_{\theta^{\mu \nu}} U^{-1} A_{\nu} U\right) T\right\} .
$$

上式指出,作用量在 Off-SHT 情况下是不变的（在 On-shell 情况下能量-动量张量也是不变 的). 从(2.15)式出发, 将得到作为 Noether 流的一系列非定域守恒流. 初看, 似可可以这样就 可得到非定域守恒流：取(2.12)与(2.15)式的差,并定义一个新的流 $\tilde{J}_{\mu}$ 的散度等于这个差, 则 这个流自然守恒. 但这样的流是平庸的, 因为 $\tilde{J}_{0}$ 在 off-shell 时为零, 而 $J_{1}$ 在 on-shell 时为 零. 为了得到非平庸流, 必须合理地从 $\tilde{J}_{\mu}$ 中拋去某些项，它们对 $\delta \mathscr{L}$ 的贡献仍旧是全散度 的形式, 并舍去由反对称张量 $\epsilon_{\mu \nu}$ 与对 $\mu, \nu$ 指标对称量相乘而恒等于零的量. 为了能明显 地作到这点, 我们将 $U(l, x)$ 对参数 $l$ 作幂级数展开, 并同以㓩文献相比较 ${ }^{[8]}$, 取

$$
U=1+e \chi^{(1)}+l^{2}\left[\chi^{(2)}+\frac{1}{2} \chi^{(1)^{2}}\right]+l^{3}\left\{\chi^{(3)}+\frac{1}{2}\left(\chi^{(1)} \chi^{(2)}+\chi^{(2)} \chi^{(1)}\right)\right\}
$$




$$
\begin{aligned}
& +l^{4}\left\{\chi^{(4)}+\frac{1}{2}\left(\chi^{(2)^{2}}+\chi^{(2)} \chi^{(1)^{2}}-\frac{1}{4} \chi^{(1)^{4}}\right)+\frac{1}{2}\left(\chi^{(1)} \chi^{(3)}+\chi^{(3)} \chi^{(1)}\right)\right\} \\
& +\cdots \cdots, \\
U^{-1} & =1-l \chi^{(1)}-l^{2}\left[\chi^{(2)}-\frac{1}{2} \chi^{(1)^{2}}\right]-l^{3}\left\{\chi^{(3)}-\frac{1}{2}\left(\chi^{(1)} \chi^{(2)}+\chi^{(2)} \chi^{(1)}\right)\right\} \\
& -l^{4}\left\{\chi^{(1)}-\frac{1}{2}\left(\chi^{(2)^{2}}-\chi^{(2)} \chi^{(1)^{2}}-\frac{1}{4} \chi^{(1)^{4}}\right)-\frac{1}{2}\left(\chi^{(1)} \chi^{(3)}+\chi^{(3)} \chi^{(1)}\right)\right\} \\
& -\cdots \cdots
\end{aligned}
$$

将(2.16),(2.17)式代入(2.9)式,并比较 $l$ 的同幂次,遂得 $\chi^{(n)}$ 所满足的方程式,例如

$$
\begin{aligned}
\partial_{1} \chi^{(1)}= & A_{0}, \\
\partial_{1} \chi^{(2)}= & -A_{1}+\frac{1}{2}\left[\chi^{(1)}, A_{0}\right], \\
\partial_{1} \chi^{(3)}= & \frac{1}{2}\left[\chi^{(2)}, A_{0}\right]-\frac{1}{2}\left[\chi^{(1)}, A_{1}\right]+\frac{1}{4}\left[\left[A_{0}, \chi^{(1)}\right], \chi^{(1)}\right]+\frac{1}{2} \chi^{(1)} A_{0} \chi^{(1)} \\
& +A_{0}, \cdots \cdots .
\end{aligned}
$$

同时,也可以将 $\delta g$ 用 $l$ 的冥次展开

$$
\delta g=\sum_{n=0}^{\infty} l^{n} \delta^{(n)} g=-g \sum_{n=0}^{\infty} l^{n} \Lambda^{(n)},
$$

求得

$$
\begin{aligned}
\Lambda^{(1)}= & {\left[\chi^{(1)}, T\right], } \\
\Lambda^{(2)}= & {\left[\chi^{(2)}, T\right]+\frac{1}{2}\left[\chi^{(1)},\left[\chi^{(1)}, T\right]\right], } \\
\Lambda^{(3)}= & {\left[\chi^{(3)}, T\right]+\frac{1}{2}\left[\chi^{(1)} T \chi^{(1)}, \chi^{(1)}\right]+\frac{1}{2}\left[\chi^{(2)},\left[\chi^{(1)}, T\right]\right] } \\
& +\frac{1}{2}\left[\chi^{(1)},\left[\chi^{(2)}, T\right]\right], \cdots \cdots .
\end{aligned}
$$

经过相当的计算, 得到由 $\delta^{(n)} \mathrm{g}$ 所引起的 $\delta^{(n)} \mathscr{\ell}$

$$
\delta \mathscr{L}=\sum_{n=0}^{\infty} l^{n} \delta^{(n)} \mathscr{L} .
$$

为(在 Off-SIHT 下)

$$
\begin{gathered}
\delta^{(1)} \mathscr{L}=\frac{1}{2} \partial_{\mu} T_{r}\left\{\left(\frac{1}{2} \epsilon^{\mu \nu}\left[\partial_{\nu} \chi^{(1)}, \chi^{(1)}\right]-\epsilon^{\mu \nu} A_{\nu}\right) T\right\}, \\
\delta^{(2)} \mathscr{L}=\frac{1}{2} \partial_{\mu} T_{r}\left\{\left(\epsilon^{\mu \nu}\left[\partial_{\nu} \chi^{(1)}, \chi^{(2)}\right]+\frac{1}{6} \epsilon^{\mu \nu}\left[\left[\partial_{\nu} \chi^{(1)}, \chi^{(1)}\right], \chi^{(1)}\right]\right.\right. \\
\left.\left.-\epsilon^{\mu \nu}\left[\mathcal{L}_{\nu}, \chi^{(1)}\right]\right) T\right\}, \\
\delta^{(3)} \mathscr{L}=\frac{1}{2} \partial_{\mu} T_{r}\left\{\epsilon ^ { \mu \nu } \left(\left[\partial_{\nu} \chi^{(1)}, \chi^{(3)}\right]+\frac{1}{2}\left[\chi^{(2)}, \partial_{\nu} \chi^{(2)}\right]+\frac{1}{2}\left[\chi^{(1)}, \partial_{\nu} \chi^{(1)}\right]\right.\right. \\
+\frac{1}{4}\left[\left[\partial_{\nu} \chi^{(1)}, \chi^{(\nu)}\right], \chi^{(2)}\right]+\frac{1}{4}\left[\left[\partial_{\nu} \chi^{(2)}, \chi^{(L)}\right], \chi^{(1)}\right]+\frac{1}{8}\left[\partial_{\nu} \chi^{(1)^{2}}, \chi^{(1)}\right]
\end{gathered}
$$




$$
\left.\left.+\left[\chi^{(2)}, A_{\nu}\right]-\frac{1}{2}\left[\chi^{(1)},\left[\chi^{(1)}, A_{\nu}\right]\right]\right) T\right\} .
$$

在得到以上诸式时，我们抛掉了形如 $\epsilon^{\mu \nu} \partial_{\mu} \partial_{\nu} \chi^{(n)}, \epsilon^{\mu \nu}\left(\partial_{\mu} \chi^{(1)} \chi^{(1)} \partial_{\nu} \chi^{(1)}+\partial_{\nu} \chi^{(1)} \chi^{(1)} \partial_{\mu} \chi^{(1)}\right)$ 以 及 $\theta^{\mu \nu}\left(\partial_{\mu} \chi^{(2)} \partial_{\nu} \chi^{(1)}+\partial_{\nu} \chi^{(2)} \partial_{\mu} \chi^{(1)}\right)$ 等恒为零的项. 为了得到守恒流, 必须回到 on-shell 的情 况, 将(2.12)式用 $l$ 的冥次展开,并分别相应地取与(2.22)-(2.24)式的差, 就可以定义一系列 新的守恒流 $J_{\mu}^{(n)}$ :

$$
\partial^{\mu} \boldsymbol{J}_{\mu^{\mu}}^{(n)}=0,
$$

其中 $J_{\mu}^{(n)}$ 的前三阶具体形式为:

$$
\begin{gathered}
J_{\mu}^{(1)}=\left[A_{\mu}, \chi^{(1)}\right]+\epsilon_{\mu \nu} A^{\nu}-\frac{1}{2} \theta_{\mu \nu}\left[\partial^{\nu} \chi^{(1)}, \chi^{(1)}\right], \\
J_{\mu}^{(2)}=\left[A_{\mu}, \chi^{(2)}\right]+\frac{1}{2}\left[\left[A_{\mu}, \chi^{(1)}\right], \chi^{(1)}\right]-c_{\mu \nu}\left[\partial_{\nu} \chi^{(1)}, \chi^{(2)}\right] \\
-\frac{1}{6} \epsilon_{\mu \nu}\left[\left[\partial^{\nu} \chi^{(1)}, \chi^{(1)}\right], \chi^{(1)}\right]+\epsilon_{\mu \nu}\left[A^{\nu}, \chi^{(1)}\right], \\
J_{\mu}^{(3)}=\left[A_{\mu}, \chi^{(3)}\right]-\frac{1}{2}\left[\chi^{(1)} A_{\mu} \chi^{(1)}, \chi^{(1)}\right]+\frac{1}{2}\left[\left[A_{\mu}, \chi^{(1)}\right], \chi^{(2)}\right] \\
+\frac{1}{2}\left[\left[A_{\mu}, \chi^{(2)}\right], \chi^{(1)}\right]-\epsilon_{\mu \nu}\left(\left[\partial^{\nu} \chi^{(1)}, \chi^{(3)}\right]+\frac{1}{2}\left[\chi^{(2)}, \partial^{\nu} \chi^{(2)}\right]\right. \\
+\frac{1}{2}\left[\chi^{(1)}, \partial^{\nu} \chi^{(1)}\right]+\frac{1}{4}\left[\left[\partial^{\nu} \chi^{(1)}, \chi^{(1)}\right], \chi^{(2)}\right]+\frac{1}{4}\left[\left[\partial^{v} \chi^{(2)}, \chi^{(1)}\right], \chi^{(1)}\right] \\
\left.+\frac{1}{8}\left[\partial^{\nu} \chi^{(1)}, \chi^{(1)^{2}}\right]+\left[\chi^{(2)}, A^{\nu}\right]-\frac{1}{2}\left[\chi^{(1)},\left[\chi^{(1)}, A^{\nu}\right]\right]\right) .
\end{gathered}
$$

(2.26)和 (2.27) 式正是文献 [8]中所提出的守恒流 (仅只度规不同). 而在我们的理论中, 这些 打恒流是作为 $H$-变换的 Noether 流而得到的,亦即由拉氏函数的 $H$-变换性质决定的，因而 可以说,与无穷多非定域守恒流相联系的“隐藏”利称性,已部分地被揭示出来了.

上述讨论全部可以在光锥坐标中进行. 引人 $\xi=x_{0}+x_{1}, \eta=x_{0}-x_{1}$ 以及 $A_{\xi}=g^{-1} \partial_{\xi} g$ 与 $A_{\eta}=g^{-1} \partial_{\eta} g$ 则线性方程为:

$$
\begin{aligned}
& \partial_{\xi} U=-\frac{l}{1+l} A_{\xi} U, \\
& \partial_{\eta} U=\frac{l}{1-l} A_{\eta} U .
\end{aligned}
$$

此时在 off-SHT 情况下有

$$
\delta \mathscr{L}=-\frac{1}{4} T_{r}\left\{A_{\xi} \partial_{\eta}\left(g^{-1} \delta g\right)+A_{\eta} \partial_{\xi}\left(g^{-1} \delta g\right)\right\} .
$$

利用平直条件与(2.29)式,有

$$
\begin{aligned}
\delta \mathscr{L}=\frac{1}{4} & T_{r}\left\{\frac{1-l^{2}}{l}\left[\partial_{\xi}\left(U^{-1} \partial_{\eta} U\right)-\partial_{\eta}\left(U^{-1} \partial_{\xi} U\right)\right] T\right. \\
& \left.-l\left[\partial_{\xi}\left(U^{-1} \eta U\right)-\partial_{\eta}\left(U^{-1} A_{\xi} U\right)\right] T\right\} .
\end{aligned}
$$

同理, 其守恒流为: 


$$
\partial_{\xi} J_{\eta}+\partial_{\eta} J_{\xi}=0
$$

其中

$$
\begin{aligned}
& J_{\xi}=U^{-1} A_{\xi} U+\frac{2}{l} U^{-1} \partial_{\xi} U=\frac{l-1}{l+1} U^{-1} A_{\xi} U, \\
& J_{\eta}=U^{-1} A_{\eta} U-\frac{2}{i} U^{-1} \partial_{\eta} U=\frac{l}{l+1} U^{-1} A_{\eta} U .
\end{aligned}
$$

它们正是光雉坐初中的通常的流的形式.

\section{三、H-变换与 Kac-Moody 代数}

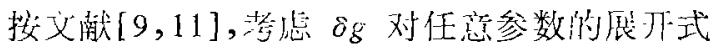

$$
\delta_{\alpha} g=\sum_{n=0}^{\infty} l^{n} \delta_{a}^{(n)} g=\sum_{n=0}^{\infty} l^{n} \delta_{u}^{(n)} g \alpha^{a}=-g U(l, g) T_{\alpha} U(l, g)^{-1},
$$

并定义算子 $Q^{(n)}=-T_{r} \int d \xi d \eta \delta_{\Delta}^{(n)} g(\xi, \eta) \frac{\delta}{\xi \xi(5, \eta)}$, 则存在关系

$$
\left[Q_{a}^{(m)}, Q_{c}^{(z)}\right]=C_{u b}^{c} Q_{c}^{(m+n)},
$$

它等价午 $[8, ?, 11]$

$$
\left[\delta_{a}^{(m)}, \delta_{b}^{(n)}\right] g=C_{a b}^{c} \delta_{c}^{(m+n)} g .
$$

这种无穷维的代数称为 Kac-Moody 代数 ${ }^{[14]}, C_{a b}^{c}$ 为有限维的群 $G$ 的结构常数.

下面将用严格的方法直接证明 (3.3)式,并揭示出 Kac-Moody 代数结构与 $H$-变换之间的 紧密联系。江琹，以下的证叫仅仅用于一个线性方程(2.29)式和 $H$-变换(3.1)式．我们仍从 标准的程序开始, 计算对易子

其中

$$
\left[\delta_{a}, \delta_{\beta}^{\prime}\right] g=\delta_{u}\left(g+\delta_{\beta}^{\prime} g\right)-\delta_{\alpha} g-\delta_{\beta}^{\prime}\left(g+\delta_{\alpha g} g\right)+\delta_{\beta}^{\prime} g,
$$

$$
\begin{aligned}
& \delta_{u} g=-g U(l, g) T_{\alpha} U(l, g)^{-1}=-g \Lambda_{\alpha}(l, g), \\
& \delta_{A}^{\prime} g=-g U\left(l^{\prime}, g\right) T_{\beta} U\left(l^{\prime}, g^{-1}=-g \Lambda_{\beta}\left(l^{\prime}, g\right),\right.
\end{aligned}
$$

以及 $T_{\alpha}=T_{a} \alpha^{u}, T_{\beta}={ }^{\prime} T_{h} \beta^{\prime \prime}$. 以下为方便起见,引入简单记号 $U=U(l, g), U^{\prime}=U\left(l^{\prime}, g\right)$, $\Lambda_{\alpha}=\Lambda_{\alpha}(l, g)$ 以及 $\Lambda_{\alpha}^{\prime}=\Lambda_{\alpha}\left(l^{\prime}, g\right)$. 由 $(3,4)$ 式得

$$
\left[\delta_{\alpha}, \delta_{\beta}^{\prime}\right] g=g\left\{\left[\Lambda_{\beta}^{\prime}, \Lambda_{\alpha}\right]+\left[\delta_{u} U^{\prime} U^{\prime-1}, \Lambda_{\beta}\right]-\left[\delta_{\beta}^{\prime} U U^{-1}, \Lambda_{\alpha}\right]\right\},
$$

其中

$$
\delta_{\alpha} U^{\prime}=U\left(l^{\prime}, g+\delta_{u} g\right)-U\left(l^{\prime}, g\right), \delta_{\beta}^{\prime} U=U\left(l, g+\delta_{\beta}^{\prime} g\right)-U(l, g) .
$$

由(2.29)式及 $A_{\xi}=g^{-1} \partial_{\xi} g$ 以及(3.5)和(3.6)式,成市。

$$
\begin{aligned}
& \delta_{\beta}^{\prime} A_{\xi}=-\frac{1}{1+l^{\prime}}\left[A_{\xi}, \Lambda_{\beta}^{\prime}\right]=\frac{1}{l^{\prime}} \partial_{\xi} \Lambda^{\prime}, \\
& \delta_{\alpha} A_{\xi}=-\frac{1}{1+l}\left[A_{\xi}, \Lambda_{\alpha}\right]=\frac{1}{l} \partial_{\xi} \Lambda .
\end{aligned}
$$

以下计算 $\delta_{\beta}^{\prime} U U^{-1}$ 与 $\delta_{\alpha} U^{\prime} U^{\prime-1}$ 的形式.

对 (2.29)式取变分, 并注意到(3.8)与(3.9)式有

$$
\partial_{\xi}\left(\delta_{\beta}^{\prime} U\right)=-\frac{l}{1+l} \cdot t_{\xi} \delta_{\beta}^{\prime} U-\frac{l}{1+l}\left(\delta_{A}^{\prime} A_{\xi}\right) U,
$$




$$
\partial_{\xi}\left(\delta_{\beta} U^{\prime}\right)=-\frac{l^{\prime}}{1+l^{\prime}} A_{\xi} \delta_{\beta} U^{\prime}-\frac{l^{\prime}}{1+l^{\prime}} \cdot\left(\delta_{\beta} A_{\xi}\right) U^{\prime},
$$

它们有形式解

$$
\begin{aligned}
& \delta_{\beta}^{\prime} U=-\frac{l}{1+l} \frac{1}{l^{\prime}} U \int_{-\infty}^{\xi} d \xi^{\prime} U^{-1} \partial_{\xi^{\prime}} \Lambda_{\beta}^{\prime} U, \\
& \delta_{\alpha} U^{\prime}=-\frac{l^{\prime}}{1+l^{\prime}} \frac{1}{l} U^{\prime} \int_{-\infty}^{\xi} d \xi^{\prime} U^{\prime-1} \partial_{\xi^{\prime}} \Lambda_{\alpha} U^{\prime} .
\end{aligned}
$$

注意

$$
U^{-1} \partial_{\xi} \Lambda_{\beta}^{\prime} U=\partial_{\xi}\left(U^{-1} \Lambda_{\beta}^{\prime} U\right)+\left(\frac{1+l^{\prime}}{1+l}\right) l U^{-1} \delta A_{\xi}^{\prime} U
$$

和类似的 $U^{\prime-1} \partial_{5} \Lambda_{\alpha} U^{\prime}$ 的表达式以及边界条件

$$
U(l, \xi \rightarrow-\infty)=1, U\left(l^{\prime}, \xi \rightarrow-\infty\right)=1,
$$

应用分部积分有

$$
\begin{aligned}
\delta_{\beta}^{\prime} U U^{-1} & =\frac{l}{l-l^{\prime}}\left(\Lambda_{\beta}^{\prime}-\Lambda_{\beta}\right), \\
\delta_{\alpha} U^{\prime} U^{\prime-1} & =\frac{l^{\prime}}{l-l^{\prime}}\left(\Lambda_{\alpha}^{\prime}-\Lambda_{\alpha}\right) .
\end{aligned}
$$

将(3.16)和(3.17)式代人(3.7)式，得

$$
\left[\delta_{\alpha}, \delta_{\beta}^{\prime}\right] g=g \frac{1}{l^{\prime}-l} \cdot\left(l^{\prime}\left[\Lambda_{\beta}^{\prime}, \Lambda_{\alpha}^{\prime}\right]-l\left[\Lambda_{\beta}, \Lambda_{\alpha}\right]\right),
$$

即

$$
\left[\delta_{o}, \delta_{\beta}^{\prime}\right]_{b^{\prime}}=\alpha^{a} \beta^{b} g\left(\frac{-1}{l^{\prime}-l}\right) C_{a b}^{c}\left(l^{\prime} \Lambda_{c}^{\prime}-l \Lambda_{c}\right),
$$

或。

$$
\left[\delta_{a}, \delta_{\beta}^{\prime}\right] g=\alpha^{a} \beta^{b} C_{a b}^{c} \frac{l^{\prime} \delta_{c}^{\prime} g-l \delta_{c} g}{l^{\prime}-l} .
$$

将(3.20)式左端用 $l$ 与 $l^{\prime}$ 展开,有

$$
\left[\delta_{\alpha}, \delta_{\beta}^{\prime}\right] g=\alpha^{a} \beta^{b} \sum_{m=0}^{\infty} \sum_{n=0}^{\infty} l^{m} l^{\prime n}\left[\delta_{u}^{(m)}, \delta_{0}^{(n)}\right] g,
$$

将(3.20)式右端也用 $l$ 与 $l^{\prime}$ 展开,有

$$
\alpha^{a} \beta^{b} C_{a b}^{c} \frac{l^{\prime} \delta^{\prime} g-l \delta_{c} g}{l^{\prime}-l}=\alpha^{a} \beta^{b} C_{a b}^{c} \sum_{k=0}^{\infty} \frac{l^{(k+1)}-l^{(k+1)}}{l^{\prime}-l} \delta_{c}^{(k)} g .
$$

因为 $\sum_{k=0}^{\infty} \frac{l^{(k+1)}-l^{(k+1)}}{l^{\prime}-l} \delta_{c}^{(k)} g=\sum_{k=0}^{\infty} \sum_{j=0}^{k} l^{\prime k-i} l^{i} \delta_{c}^{(k)} g=\sum_{m=0}^{\infty} \sum_{n=0}^{\infty} l^{m} l^{\prime n} \delta_{c}^{(m+n)} g$, 故最后有

$$
\left[\delta_{a}^{(m)}, \delta_{b}^{(n)}\right] g=C_{a b}^{c} \delta_{c}^{(m+n)} g,
$$

它正是 Kac-Moody 代数结构. 应当指出的是, 解出 $\delta_{\theta}^{\prime} U U^{-1}$ 的具体形式是不必要的, 实际上, 将 $\delta_{\beta}^{\prime} U U^{-1}$ 直接对 $\xi$ 微商, 并考虑到线性方程(2.29)以及(3.8)式,有

$$
\partial_{\xi}\left(\delta_{\beta}^{\prime} U U^{-1}\right)=-\frac{l}{1+l}\left(\frac{1}{l^{\prime}} \partial_{\xi} \Lambda_{\beta}^{\prime}+\left[A_{\xi}, \delta_{\beta}^{\prime} U U^{-1}\right]\right) \text {. }
$$


另一方面,由(2.29)式得

$$
\partial_{\xi} A_{\beta}^{\prime}=-\frac{l^{\prime}}{1+l^{\prime}}\left[A_{\xi}, \Lambda_{\beta}^{\prime}\right]
$$

利用(3.8)式，上式可化为:

$$
\partial_{\xi}\left\{\frac{l}{l-l^{\prime}}\left(\Lambda_{\beta}^{\prime}-\Lambda_{\beta}\right)\right\}=-\frac{l}{1+l}\left\{\frac{1}{l^{\prime}} \partial_{\xi} \Lambda_{\beta}^{\prime}+\left[A_{\xi}, \frac{l}{l-l^{\prime}}\left(\Lambda_{\beta}^{\prime}-\Lambda_{\beta}\right)\right]\right\},
$$

比较(3.23)与(3.25)式, 方程全同, 而且由于当 $\xi \rightarrow-\infty$ 时,

$$
\delta_{\beta}^{\prime} U U^{-1} \rightarrow 0, \Lambda_{\beta}^{\prime}-\Lambda_{\beta} \rightarrow 0,
$$

故同样有(3.16)与(3.17)式成立. 同样需要指出的是, 由(3.16)与(3.17)式, 当取 $l^{\prime} \rightarrow l$ 时成 立

$$
\delta_{\beta} U U^{-1}=-l \frac{d \Lambda_{\beta}}{d l}=-\left[l \frac{d U}{d l} U^{-1}, \Lambda_{\beta}\right] .
$$

它正是文献[11]中的结果. 上述证明, 说明关于 $l^{\prime} \rightarrow l$ 取极限的过程是成立的. 因此, 为了 简捷与方便, 在以后的讨论中可以沿用文献[11]的推导, 但是应当理解为其极限过程已得到了 证明,或者说那里的运算已经代表了上述的论证.

\section{四、自对偶杨-Mills 方程中的 $H$-变换、Kac-Moody 代数与 Bäcklund 变换}

利用杨-Mills 场的 $J$-形式 ${ }^{[15,16]}$. 按文献 $[15,16]$ 我们写下自对偶杨-Mills 场方程的一系 列公式:

自对偶条件

$$
F_{\mu \nu}=\partial_{\mu} A_{\nu}-\partial_{\nu} A_{\mu}+\left[A_{\mu}, A_{\nu}\right] .
$$

$$
F_{\mu \nu}=\frac{1}{2} \epsilon_{\mu \nu \rho \sigma} F_{\rho \sigma} .
$$

当引人复坐标后，

$$
\begin{array}{ll}
\sqrt{2} y=x_{1}+i x_{2}, & \sqrt{2} \bar{y}=x_{1}-i x_{2}, \\
\sqrt{2} z=x_{3}-i x_{4}, & \sqrt{2} \bar{z}=x_{3}+i x_{4},
\end{array}
$$

则(4.2)式变为:

$$
\begin{aligned}
& F_{Y Z}=F_{\bar{Y} \bar{Z}}=0, \\
& F_{Y \bar{Y}}+F_{Z \bar{Z}}=0 .
\end{aligned}
$$

按杨 ${ }^{[16]}$ 引

$$
\begin{aligned}
& A_{Y}=D^{-1} D_{1}, A_{Z}=D^{-1} D_{Z}, \\
& A_{\bar{Y}}=\bar{D}^{-1} \bar{D}_{\bar{Y}}, A_{\bar{Z}}=\bar{D}^{-1} \bar{D}_{\bar{Z}},
\end{aligned}
$$

其中 $D_{Y}=\partial D / \partial y$ 等等, 且 $\operatorname{det} D=\operatorname{det} \widetilde{D}=1$.

按文献 [15]定义矩阵 $J$

$$
J=D \bar{D}^{-1}, \quad \text { det } J=1 .
$$

于是自对偶场方程棸为:

$$
\left(J^{-1} J_{Y}\right)_{\bar{Y}}+\left(J^{-1} J_{Z}\right)_{\bar{Z}}=0,
$$


或

$$
\left(J J_{\bar{Y}}^{-1}\right)_{Y^{\prime}}+\left(J J_{\bar{Z}}^{-1}\right)_{Z}=0 \text {. }
$$

引人

$$
\begin{array}{ll}
B_{\mathrm{Y}}=J^{-1} J_{Y^{\prime}}, & B_{Z}=J^{-1} J_{Z}, \\
B_{\bar{Y}}=J J_{\bar{Z}^{\prime}}^{-1}, & B_{\bar{Z}}=J J_{\bar{Z}}^{-1} .
\end{array}
$$

从场方程出发, 线性方程为 ${ }^{[7]}$ :

$$
\begin{gathered}
\partial_{\bar{Z}} \psi=\lambda \mathscr{\mathscr { Y }} \psi \\
-\partial_{\overline{\mathrm{Y}}} \psi=\lambda \mathscr{D}_{\mathrm{Z}} \psi, \quad \mathscr{D}_{u}=\partial_{u}+B_{u} .
\end{gathered}
$$

其可积条件导致场方程

$$
\left(B_{Y}\right)_{\bar{Y}}+\left(B_{Z}\right)_{\bar{z}}=0
$$

与

$$
\left(B_{\bar{Y}}\right)_{Y}+\left(B_{\bar{Z}}\right)_{Z}=0 .
$$

对于 $S L(2, c)$ 自对偶情况, 文献 $[7,15]$ 引 入 Bäcklund 变换

$$
\begin{aligned}
& J^{\prime-1} J_{Y}^{\prime}-J^{-1} J_{Y}=\lambda^{\prime \prime}\left(J^{-1} J^{\prime}\right), \\
& J^{\prime-1} J_{Z}^{\prime}-J^{-1} J_{L}=-\lambda^{\prime \prime}\left(J^{-1} J^{\prime}\right)_{\bar{Y}},
\end{aligned}
$$

$\lambda^{\prime \prime}$ 为任意参数. 如果限制 $j^{\prime}$ 仍为厄米, 则相当于必须存在一些限制条件

$$
\lambda=e^{i \alpha}, J^{\prime} J^{-1}-J J^{\prime-1}=B I,
$$

其中 $\alpha, \beta$ 为实数, $I$ 为单位矩阵.

引入对于 $J$ 的 $H$-变换

$$
J^{-1} \delta_{\alpha} J=-\Psi(\lambda, J) T_{\alpha} \Psi(\lambda, J)^{-1}=-\Lambda_{\alpha},
$$

戋

$$
\delta J=-J \Lambda_{a} .
$$

以下我们从场方程出发讨论. 首先易证明在变换(4.19)式下场方程不变. 利用 (4.19) 和 (4.10)式可证

$$
\delta B_{Y}=D_{Y}\left(J^{-1} \ddot{u} J\right)
$$

同理

$$
\delta B_{\hat{\mathbf{Y}}}=D_{\overline{\mathbf{Y}}}\left(J^{-1} \delta J\right),
$$

其中

$$
D_{u}=\partial_{u}+\left[J^{-1} J_{u},\right]
$$

于是场方程 (4.14)式的变化为:

$$
\partial_{\bar{Y}}\left(\delta B_{Y}\right)+\partial_{\bar{Z}}\left(\delta B_{Z}\right)=\left(D_{Y} \partial_{\ddot{Y}}+D_{Z} \partial_{\bar{Z}}\right)\left(J^{-1} \delta J\right) .
$$

由变换(4.19)式及线性化方程 (4.12)及(4.13) 式可证明

$$
\begin{aligned}
& \partial_{\bar{Y}}\left(J^{-1} \delta J\right)=-\lambda D_{Z}\left(J^{-1} \delta J\right), \\
& \partial_{\bar{Z}}\left(J^{-1} \delta J\right)=\lambda D_{Y}\left(J^{-1} \delta J\right) .
\end{aligned}
$$

注意到 $\partial_{Y} B_{Z}-\partial_{Z} B_{Y}+\left[B_{Y}, B_{Z}\right]=0$, 痟

$$
\partial_{\bar{Y}} \delta B_{Y}+\partial_{\bar{Z}} \delta B_{Z}=0 .
$$

洲理可证

$$
\partial_{Y} s B_{Y}+\partial_{L} v B_{A}=0
$$


所以，与前几节类似,变换 (4.19)式能保持运动方程不变. 由于于

$$
J^{\prime-1} J_{Y}^{\prime}-J^{-1} J_{Y}=B_{Y}^{\prime}-B_{Y}=\delta B_{Y},
$$

以及

$$
D_{\mathrm{Y}}\left(J^{-1} \varepsilon J\right)=D_{\mathrm{Y}}\left(J^{-1} J^{\prime}\right)
$$

于是(4.21) 式可写为:

$$
J^{\prime-1} J_{Y}^{\prime}-J^{-1} J_{Y}=D_{1}\left(J^{-1} \delta J\right) .
$$

将(4.26)式代入(4.31)式,并考虑到(4.30)式有

$$
J^{\prime-1} J_{Y}^{\prime}-J^{-1} J_{Y}=\frac{1}{\lambda}\left(J^{-1} J^{\prime}\right)_{\bar{z}}
$$

令

$$
\lambda^{\prime \prime}=1 / \lambda,
$$

则(4.32)式恰是 Bäcklund 变换式(4.16). 同理可得(4.17)式.

但是我们看到, 在变换 (5.19)式之后， $J^{\prime}=J+\delta J$ 与 $J$ 有些不同的性质：如果 $J$ 是厄米 的, $J^{\prime}$ 不再保持厄米. 这意味着, 如果 $J$ 是在 $S U(2)$, 则 $J^{\prime}$ 将越出这个范围(例如 $S L(2$, C)). 这种现象在杨-Mills 理论中非常普遍, 在多个磁单极的解的问题里是用规范变换再变回 $S U(2)$ 范围内 ${ }^{[15]}$. 但目前我们尚不知道在本问题中的规范变换形式.

最后, 我们从线性化方程(4.12),(4.13)式及变换(4.19)式出发, 讨论其 kac-Moody 代数结 构问题。此时, (4.19) 式中的 $J$ 相当于 Chiral 模型中 $g$ 的地位. 按照标准的计算, 必须考虑

$$
\left[\delta_{\alpha}, \delta_{\beta}^{\prime}\right] J=\delta_{\alpha}\left(J+\delta_{\beta}^{\prime} J\right)-\delta_{\alpha} J-\delta_{\beta}^{\prime}\left(J+\delta_{\alpha} J\right)+\delta_{\beta}^{\prime} J .
$$

引入与第三当监中(3.5)与(3.6)式相同的记号, 只是注意将 $g \rightarrow J, U \rightarrow \psi$, 则有

$$
\left[\delta_{\alpha}, \delta_{\beta}^{\prime}\right] J=J\left\{\left[\Lambda_{\beta}^{\prime}, \Lambda_{\alpha}\right]+\left[\delta_{\alpha} \psi^{\prime} \psi^{\prime-1}, \Lambda_{\beta}\right]-\left[\delta_{\beta}^{\prime} \psi \psi^{-1}, \Lambda_{\alpha}\right]\right\} .
$$

重复第三节的讨论, 只需证明

$$
\left[\delta_{\alpha}, \delta_{\beta}^{\prime}\right] J=\alpha^{i} \beta^{b} J C_{a b}^{c} \cdot(-1) \frac{\lambda^{\prime} \Lambda_{c}^{\prime}-\lambda \Lambda_{c}}{\lambda^{\prime}-\lambda},
$$

就证明了 Kac-Moody 代数的结松. 以下找们从(4.12),(4.13)式以及(4.19)式证明(4.36)式成 立.

首先, 由(4.12)，(4.13)式易证成立

$$
\partial_{\tilde{y}} \Lambda_{\alpha}=-\lambda D_{Z} \Lambda_{\alpha},
$$

和

以及

$$
\partial_{\bar{z}} \Lambda_{\alpha}=\lambda D_{Y} \Lambda_{\alpha}
$$

$$
\begin{aligned}
& \partial_{\bar{Y}} \Lambda_{\alpha}^{\prime}=-\lambda^{\prime} D_{z} \Lambda_{\alpha}^{\prime}, \\
& \partial_{\bar{Z}} \Lambda_{a}^{\prime}=\lambda^{\prime} D_{Y} \Lambda_{a}^{\prime} .
\end{aligned}
$$

还成立

$$
\begin{aligned}
& \delta_{\alpha} B_{Y}=-\frac{1}{\lambda} \partial_{\bar{Z}} \Lambda_{\alpha}, \quad \delta_{\alpha}^{\prime} B_{Y}=-\frac{1}{\lambda^{\prime}} \partial_{\bar{Z}} A_{\alpha}^{\prime}, \\
& \delta_{\alpha} B_{Z}=\frac{1}{\lambda} \partial_{\bar{Y}} A_{\alpha}, \quad \delta_{\alpha}^{\prime} B_{Z}=\frac{1}{\lambda^{\prime}} \partial_{\bar{Y}} A_{\alpha}^{\prime} .
\end{aligned}
$$

由(4.12)与(4.41)式推得 


$$
\partial_{\bar{Z}}\left(\delta_{\beta}^{\prime} \psi \psi^{-1}\right)=\lambda\left\{\left(\partial_{Y} \delta_{\beta}^{\prime} \psi\right) \psi^{-1}-\frac{1}{\lambda^{\prime}} \partial_{\bar{Z}} \Lambda_{\beta}^{\prime}+B_{Y}\left(\delta_{\beta}^{\prime} \psi\right) \psi^{-1}\right\}+\delta_{\beta}^{\prime} \psi \partial_{\bar{Z}} \psi^{-1} .
$$

再代人(4.12)式有

$$
\partial_{\bar{Z}}\left(\delta_{\beta}^{\prime} \psi \psi^{-1}\right)=\lambda\left\{\partial_{Y}\left(\delta_{\beta}^{\prime} \phi \psi^{-1}\right)-\frac{1}{\lambda^{\prime}} \partial_{\bar{Z}} \Lambda_{\beta}^{\prime}+\left[B_{Y}, \delta_{\beta}^{\prime} \psi \psi^{-1}\right]\right\} .
$$

另一方面, 取( 4.40$)$ 与(4.38)式的差,并作变形后有

$$
\partial_{\bar{Z}}\left(\Lambda_{\beta}^{\prime}-\Lambda_{\beta}\right)=\lambda\left\{\partial_{Y}\left(\Lambda_{\beta}^{\prime}-\Lambda_{\beta}\right)+\left[B_{Y}, \Lambda_{\beta}^{\prime}-\Lambda_{\beta}\right]+\frac{\lambda^{\prime}-\lambda}{\lambda} D_{Y} \Lambda_{\beta}^{\prime}\right\},
$$

利用(4.40)式, 有

$$
\begin{gathered}
\partial_{\bar{Z}}\left[\left(\frac{\lambda}{\lambda-\lambda^{\prime}}\right)\left(\Lambda_{\beta}^{\prime}-\Lambda_{\beta}\right)\right]=\lambda\left\{\partial_{Y}\left[\left(\frac{\lambda}{\lambda-\lambda^{\prime}}\right) \cdot\left(\Lambda_{\beta}^{\prime}-\Lambda_{\beta}\right)\right]\right. \\
\left.+\left[B_{Y},\left(\frac{\lambda}{\lambda-\lambda^{\prime}}\right)\left(\Lambda_{\beta}^{\prime}-\Lambda_{\beta}\right)\right]-\frac{1}{\lambda^{\prime}} \partial_{\bar{Z}} \Lambda_{\beta}^{\prime}\right\} .
\end{gathered}
$$

同理,使用另一组线性方程和(4.42)式有

$$
\partial_{\overline{\mathbf{Y}}}\left(\delta_{\beta}^{\prime} \psi \psi^{-1}\right)=-\lambda\left\{\partial_{Z}\left(\delta_{\beta}^{\prime} \psi \psi^{-1}\right)+\frac{1}{\lambda^{\prime}} \partial_{\overline{\mathrm{Y}}} \Lambda_{\beta}^{\prime}+\left[B_{Z}, \delta_{\beta}^{\prime} \psi \psi^{-1}\right]\right\}
$$

和

$$
\begin{gathered}
\partial_{\overline{\mathrm{Y}}}\left[\left(\frac{\lambda}{\lambda-\lambda^{\prime}}\right)\left(\Lambda_{\beta}^{\prime}-\Lambda_{\beta}\right)\right]=-\lambda\left\{\partial _ { Z } \left[\left(\frac{\lambda}{\lambda \cdot \lambda^{\prime}}\right)\left(\Lambda_{\beta}^{\prime}-\Lambda_{\beta}\right)+\frac{1}{\lambda^{\prime}} \partial_{\overline{\mathrm{Y}}} \Lambda_{\beta}^{\prime}\right.\right. \\
\left.+\left[B_{\%}, \frac{\lambda}{\lambda-\lambda^{\prime}}\left(\Lambda_{\beta}^{\prime}-\Lambda_{\beta}\right)\right]\right\} .
\end{gathered}
$$

由边界条件

$$
\phi(l, x \rightarrow-\infty)=\psi\left(l^{\prime}, x \rightarrow-\infty\right)=1,
$$

比较(4.43)与(4.44)式, (4.45)与(4.46)式推得

$$
\delta_{\beta}^{\prime} \phi \psi^{-1}=\frac{\lambda}{\lambda-\lambda^{\prime}}\left(\Lambda_{\beta}^{\prime}-\Lambda_{\beta}\right) .
$$

对 $\delta_{\alpha} U^{\prime} U^{\prime-1}$ 也可推出类似的等式:

$$
\delta_{\alpha} \psi^{\prime} \psi^{\prime-1}=\frac{\lambda^{\prime}}{\lambda-\lambda^{\prime}}\left(\Lambda_{a}^{\prime}-\Lambda_{\alpha}\right) .
$$

将(4.48)与(4.49)式代入(4.35)式后, 遂得(4.36)式,重复以前的讨论得到在自对偶杨-Mills 场 情况下的 Kac-Moody 代数

$$
\left[\delta_{a}^{(m)}, \delta_{b}^{(n)}\right] J=C_{a b}^{c} \delta_{c}^{(m+n)} J
$$

\section{五、结 语}

我们引入了 $H$-变换, 讨论了无穷多非定域守恒流作为 Noether 流的问题, 给出了用场变 量写出各阶流的系统方案, 并从线性方程和 $H$-变换出发, 严格证明了存在 Kac-Moody 代数结 构. 作为这种讨论的推广, 着重讨论了自对偶杨-Mills 场情况, 除了存在同样的代数结构之 外,我们还发现了其 Bäcklund 变换与 $H$-变换的密切联系. 同时, 我们还要指出, 所有的讨论 全部可以使用于超手征场中去,尤其在超场表述中更为方便 ${ }^{[17]}$. 这一工作已经完成,但限于篇 幅, 将在另文讨论. 
因此，H-变换必定有更深刻的几何与物理上的原因. 尤其是，如本文所指出们，它能将 前阶段还分别看待的无穷多守恒流、Bäcklund 变换以及 Kac-Moody 代数结构密切地联系起 来，这是件非常有兴趣的事。探讨其本源以及如何将上述思想推广到二维统计模型 ${ }^{[18]}$ 以及二 维对称的引力理论 ${ }^{[19,20]}$ 将是更有兴趣的工作.

作者侯伯宇、吴詠时、葛墨林在纽约州立大学石溪分校工作期间，杨振宁和最华桐先生给 予了多方面的帮助和指导; 周光召和董明德先生也同我们进行过有益的讨论,这里一并表示感 谢.

\section{参考文献}

[1 ] Lüscher, M. \& Pohlmeyer, K., Nucl. Phys., B137(1978), 46.

[2] Brezin. E., Itzykson, C, et a]., Phys. Lett, 82B (1979), 442.

[3] De Vega, II. J., ibid., 87B(1979), 233.

[4] Eichenherr, H. \& Forger, M., Nucl. Phys., B15a (1979), 381.

[5] Ogielski, A. T., Phys. Rev., D21(1980), 406.

[6] Zichos, C., ibid., D21(1980), 3462.

[7] L-L. Chau Wang, in Proceedings of the Guangzhou Conference on Theoretical Particle Phy;ics, Jan., 5-14, 1980.

[8] Dolan, L. \& Roos, A.. Phys. Rev., D22(1980), 2018.

[9] Dolan, L., Rockefeller preprint, RU81/B5, 1981.

[10] Hou, B-Y, Yale preprint, YTP80-29 1980; Hou, B-Y Ge, M-L. \& Wu, Y-S., Phys. Rev., D24 (1981), 2238.

[11] Ge. M-L. \& Wu. Y-S., Phys. Lett., B108(1982), 411.

[12] Chau, L-L., Ge, M-1. \& Wu, Y.S., Phys. Rev., D25(1982), 1086.

[13] Hou, B-Y., Duan, Y.S. \& Ge, M.L., Scientia Sinica, 21(1978), 446.

[14] Kac, V. G., Math USSR, 2(1968), 6:127; Moody,R., J. Algebra, 10(1968), 211. Lepowsky, J. \& Wilson, R. L., Comm. Math. Phys., 62(1978), 43-53.

[15] Chau, L-L. Wang, Lectures at the 18th Winter School of Theoretical Physics, Karpacz, Poland, Feb. 8.March 4. 1981.

[16] Yang, C. N., Phys. Rev. Lett., 38(1977), 1377.

[17] Chau, L-L., Ge, M-I. \& Wr. Y-S., Phys. Rev., D25(1982), 1080.

[18] Lowenstein, J. H., Dynamical Generation of Mass in a Two-Dimensional Model, preprint of New York University.

[19] Ernst, F. J., Phys. Rev., 167(1967), 1175.

[20] Belinski1, V. A. \& Zakharov, V. E., $2 h$. Eksp. Teor. Fiz, 75(1978), 1955. 